\title{
A COMPLETE, MULTICOLOUR SURVEY OF ABSOLUTE PROPER MOTIONS IN THE ANTICENTRE DIRECTION AT INTERMEDIATE LATITUDE: KINEMATICS OF THE GALAXY'S STELLAR POPULATIONS
}

\author{
D.K. OJHA, O. BIENAYMÉ and A.C. ROBIN \\ Observatoire de Besançon \\ BP 1615, F-25010 Besançon Cedex \\ France
}

\begin{abstract}
We present the observational results of UBV photometry and absolute proper motions in the anticentre direction at intermediate latitude $\left(l=167.5^{\circ}, b=47.4^{\circ}\right)$ for an 8.6 square degree field. The sample is complete down to $\mathrm{V}=17$, and the accuracy of the proper motions is better than 0.25 per century for stars brighter than $\mathrm{V}=16$. It is attempted here to make an investigation of the structure and kinematics of the Galaxy from absolute proper motions. Details of the present work can be found elsewhere (Ojha et al. 1993).
\end{abstract}

\section{Velocity Distribution}

We have chosen a subsample of the stars in $0.3<(B-V)<0.9$ colour range (mainly $F$ and G-type stars). The photometric distance of each star has been derived using a $M_{v}$ and B-V relation and a correction for vertical metallicity gradient is applied on distance measurements. The measured proper motions have been directly converted to $(\mathrm{U}+\mathrm{W})$ and $\mathrm{V}$ velocities.

\section{Results}

\subsection{RADIAL GRADIENT OF VELOCITY DISPERSION $\left(\partial \ln \sigma_{U+W}^{2} / \partial r\right)$}

We have compared our data set with the similar data in the galactic centre at intermediate latitude $\left(l=3^{\circ}, b=47^{\circ}\right.$ ) by Bienayme et al. (1992). The same selection criterion in B-V colour (as described above) has been applied to these data. By comparing the velocity dispersion $\left(\sigma_{\mathrm{U}+\mathrm{W}}\right)$ in the two fields (Fig. 1a), our estimation of the gradient of velocity dispersion $\left(\partial \ln \sigma_{U+W}^{2} / \partial r\right)$ with galactic radius comes out to be $-0.21 \pm 0.1 \mathrm{kpc}^{-1}$.

\subsection{THE SCALE LENGTH OF OLD DISK}

By comparing the number ratio of stars between the two distributions (Fig. 1b), we deduce the scale length of old disk which is found to be 2 to $2.5 \mathrm{kpc}$. 

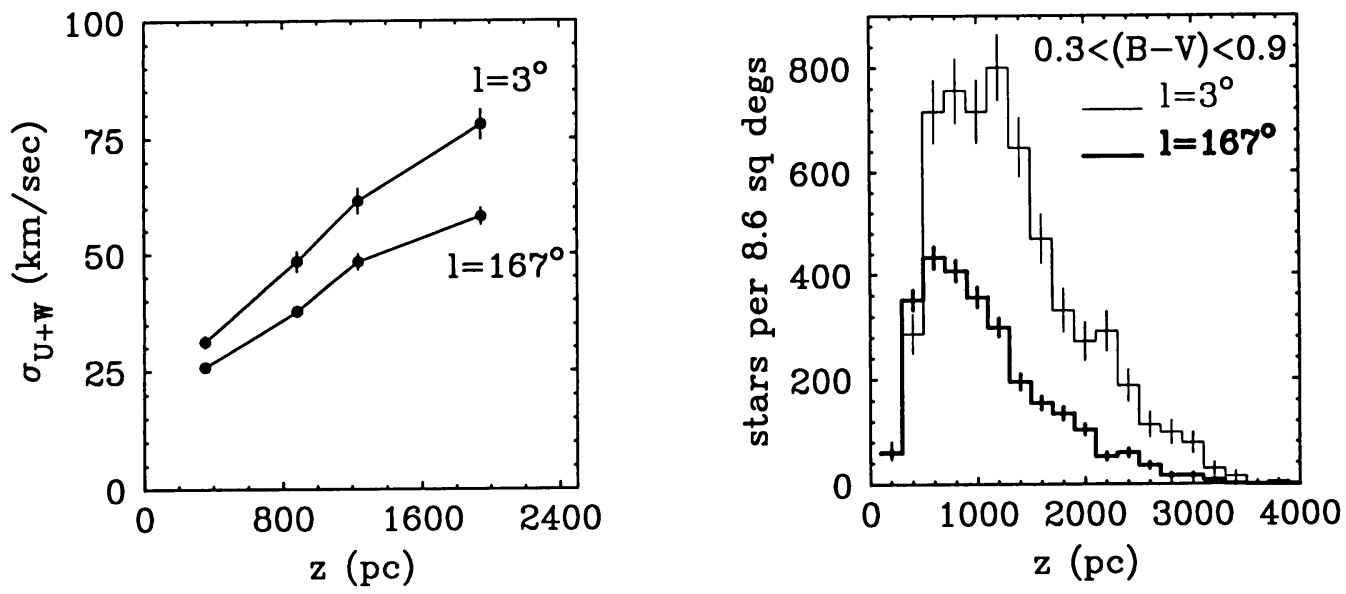

Figure 1. (a) Plot of $\sigma_{\mathrm{U}+\mathrm{w}}$ as a function of $\mathrm{z}$ for two surveys (as described in the text). (b) Distribution of $\mathbf{z}$ for two data sets.

\subsection{OLD DISK AND THICK DISK KINEMATICS}

The algorithm SEM (Stochastic Estimation Maximization) is used for the deconvolution of multivariate gaussian distributions using a maximum likelihood criterion. The kinematical parameters of the thin disk have been derived up to a distance $z=1750 \mathrm{pc}$. The mean velocities for this population are $(\langle U+W\rangle,\langle V\rangle)=(1 \pm 4,-14 \pm 2) \mathrm{km} / \mathrm{s}^{-1}$ with velocity dispersions $\left(\sigma_{U+W}, \sigma_{V}\right)=(43 \pm 2,34 \pm 1) \mathrm{km} / \mathrm{s}^{-1}$.

It is found that the thick disk has an asymmetric drift of $57 \pm 4 \mathrm{~km} / \mathrm{s}^{-1}$ with respect to LSR. This strengthens the hypothesis of a rapidly rotating population $\left(V_{\text {rot }}=173 \pm 4 \mathrm{~km} / \mathrm{s}^{-1}\right)$. The mean value of $\mathrm{V}$ velocity dispersion $\left(\sigma_{v}\right)$ is $60 \pm 3 / \mathrm{km} \mathrm{s}^{-1}$ for this population. The 3 gaussian populations representing the thin disk, the thick disk and the halo are overplotted on the $\mathrm{V}$ velocity histogram in Fig. 2 for the distance interval $1500<\mathrm{z}<2500$ pc.

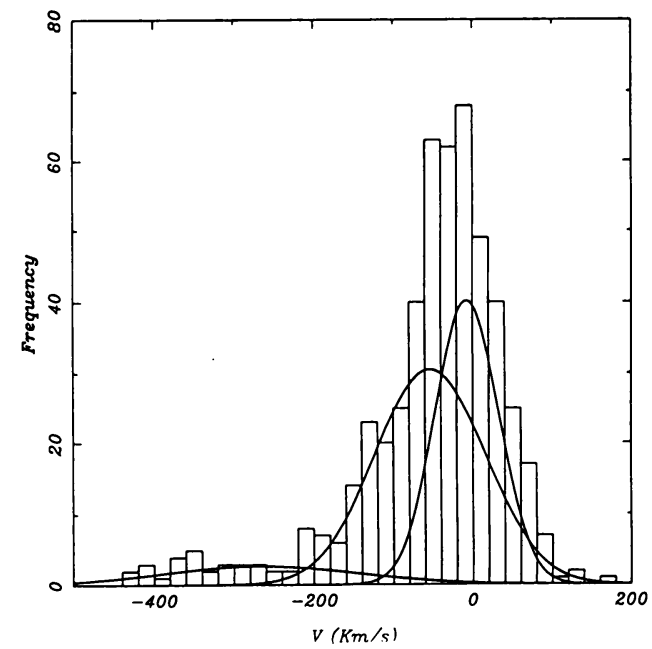

Figure 2. Histogram of the $\mathrm{V}$ velocity for stars $1500<\mathrm{z}<2500 \mathrm{pc}$. The 3 gaussian components comespond to the thin disk, thick disk and halo populations. 


\section{Conclusions}

This result confirms that the disk has a relatively short scale length of 2 to $2.5 \mathrm{kpc}$. A radial gradient of velocity dispersion in $U+W$ is found and we confirm an asymmetric drift of the order of $60 \mathrm{~km} / \mathrm{s}^{-1}$ and $a \sigma_{v}$ of $60 \mathrm{~km} / \mathrm{s}^{-1}$ for the thick disk population.

\section{References}

Bienaymé, O., Mohan, V., Crézé, M., Considère, S. and Robin, A.C., 1992. Astron. Astrophys., 253, 389.

Ojha, D.K., Bienaymé, O., Robin, A.C. and Mohan, V., 1993. Astron. Astrophys., submitted. 